\title{
Le lieu de la «bonne mort » pour les migrants guinéens et bangladais au Portugal
}

The Place of "Good Death" for the Migrants from Bangladesh and from GuineaBissau in Portugal

El lugar de la «buena muerte» para los migrantes guieneses y bangladeshís en Portugal

\section{Clara Saraiva et José Mapril}

Traducteur : Florence Lévi

\section{Q OpenEdition}

\section{Édition électronique}

URL : https://journals.openedition.org/remi/6020

DOI : $10.4000 /$ remi.6020

ISSN : $1777-5418$

Éditeur

Université de Poitiers

\section{Édition imprimée}

Date de publication : 1 septembre 2012

Pagination : $51-70$

ISBN : 979-10-90426-05-4

ISSN : 0765-0752

Référence électronique

Clara Saraiva et José Mapril, « Le lieu de la «bonne mort » pour les migrants guinéens et bangladais au Portugal », Revue européenne des migrations internationales [En ligne], vol. 28 - n³ | 2012, mis en ligne le 01 septembre 2015, consulté le 14 avril 2022. URL : http://journals.openedition.org/remi/6020 ; DOI : https://doi.org/10.4000/remi.6020 


\section{Le lieu de la " bonne mort " pour les migrants guinéens et bangladais au Portugal}

\section{Clara Saraiva' ${ }^{1}$ et José Mapril ${ }^{2}$}

Au cours des dernières décennies, les dimensions transnationales des phénomènes migratoires ont eu une importance théorique et ethnographique croissante. En utilisant le terme de "transnational " dans nos propres recherches nous nous référons au concept élaboré par Basch, Schiller et Blanc, entre 1992 et 1997, lequel renvoie aux liens multiples et permanents instaurés entre le pays " d'origine " et le pays d' "accueil ", sous leurs facettes économiques, politiques et culturelles. L'une des conséquences de ce changement d'interprétation a été de constater que de tels liens - réels et imaginaires - s'accompagnent fréquemment d'une construction sociale et symbolique d'espaces, de lieux d'appartenance. Comme l'ont fait remarquer Olwig (2007) et Geertz (1996), la mobilité s'est accompagnée de processus quotidiens de production de lieux.

En contexte de migration transnationale, ces " mondes autour de nous " se manifestent et se produisent, à la faveur de transferts de capitaux et de biens, dans l'accomplissement de rituels et de cérémonies spécifiques ou dans la reproduction de discours nationalistes. Ainsi, c'est la construction d'une certaine familiarité dans un monde de flux et de déplacements multiples qui est en cause.

Dans les études sur la migration, la mort est un bon exemple de circulation d'univers symboliques où la notion de processus est fondamentale pour comprendre le maintien de la relation avec l'espace d'origine. Tout au long de cet article, nous chercherons à montrer que la mort est une bonne métaphore pour penser cette production de lieux et d'espaces d'appartenance en contexte transnational. Pour ce faire, nous nous appuierons sur deux études de cas : les migrants du Bangladesh et de Guinée-Bissau au Portugal ${ }^{3}$.

Les conceptions de la mort et du mourir ont été un sujet d'étude pour nombre d'anthropologues; des plus classiques comme Frazer, Hertz et Evans-Pritchard jusqu'aux plus

1 Chercheure, Instituto de Investigação Científica Tropical (IICT) et Centro em Rede de Investigação em Antropologia (CRIA), Université Nationale de Lisbonne (UNL), CRIA-FCSH/UNL, Avenida de Berna, 26c, 1069-061 Lisboa, Portugal ; clarasaraiva@fcsh.unl.pt

2 Chercheur, Centro em Rede de Investigação em Antropologia CRIA-IUL, Avenida da forças armadas, Ed.Sala 2n7, 1649-026, Lisboa, Portugal ; jmapril@gmail.com

L'article a été traduit du portugais au français par Florence Levy.

3 Le travail de terrain, au Portugal, en Guinée et au Bangladesh, a consisté en observation participante, réalisation d'entretiens en profondeur, histoires de vie et recherche d'archives et de bibliographie. 
contemporains comme Gable et Boeck. Pour João de Pina Cabral (1984), les différentes approches incluent le symbolisme des divers éléments cérémoniels étudiés jusqu'aux rituels funéraires perçus comme rites de passage. Dans notre article, nous mettrons d'abord l'accent sur la relation entre d'une part, les cérémonies funèbres et d'autre part, la reproduction et la symbolisation des lieux d'appartenance ; lieux qui comportent, entre autres, une "communauté " locale 4 , un espace de diaspora, voire un État-nation. Nous analyserons ensuite la manière dont la notion d'appartenance à un lieu est en relation avec l'idée de la " bonne mort ".

Un des travaux les plus emblématiques sur la mort est certainement le livre coordonné par Maurice Bloch et Jonathan Parry (1982) Death and the Regeneration of Life dans lequel les différents auteurs s'intéressent à la présence de symboles de fertilité et de renaissance dans les rituels funéraires. Ainsi, parmi les diverses thématiques abordées pour analyser les conceptions de la mort et les rituels funéraires dans des contextes ethnographiques, celles qui dominent sont précisément celles de la relation entre les lieux et les cérémonies funèbres et celle de la notion de "bonne mort ". Dans le dernier texte de la compilation, Bloch reprend en partie une problématique développée dans sa principale œuvre ethnographique Placing the Dead (1971), où il attire l'attention sur la relation entre les rituels funéraires et I'identification au pays. À partir de sa recherche sur les Merina de Madagascar, il soutient que la mort et les rituels funéraires impliquent deux enterrements. Un premier, à proximité du lieu où la personne est morte, afin de laver le cadavre des substances impures qui le composent. Deux ans plus tard, le corps est déplacé pour être à nouveau enterré, mais cette fois-ci dans le caveau où se trouvent ses ancêtres : la sépulture et le groupe de parents étant équivalents, être enterré dans la même terre signifie une réunion avec les parents passés, présents et futurs. Cet enterrement en terre ancestrale constitue une célébration de l'union avec les réseaux familiaux et une victoire sur la division et la séparation qu'implique la vie quotidienne. Ce second enterrement, connu aussi sous le nom de famadihana, repositionne les Merina qui sont morts loin de leur terre ancestrale dans celle qu'ils croient être la terre de leurs ancêtres. Une société associée à un ordre immuable, reposant sur la parenté et le territoire qui font contrepoids à la précarité de la vie quotidienne et qui, de la sorte, apporte une continuité à l'existence.

Philippe de Boeck (2008) s'est intéressé aux danses avec les morts parmi des jeunes adultes à Kinshasa. Il affirme que la mort est vue comme un moyen de gestion des tensions sociales. À travers elle est critiqué le rôle des plus âgés - les adultes -, une génération qui a renoncé à lutter pour les droits et les conditions des plus jeunes. Dans ce contexte, les rituels funéraires cherchent à repositionner les environnements moraux de la vie publique urbaine : ce qu'on désigne comme "l'ordre du désordre ". Les jeunes aspirent à un nouveau rôle dans l'ordre postcolonial où leur place est instable, sujette à l'insécurité et à des conditions sociales extrêmes où la mort est une menace constante. Danser avec les morts est une manière de contester cet ordre. C'est aussi la mort qui est manipulée en tant que forme de contestation de cet ordre lui-même qui l'a créée, de contestation de la crise, qui a fait naître le vide moral dans la société, dans lequel sont irrémédiablement plongés les jeunes.

Cette relation entre la mort, les cérémonies funèbres et les lieux d'appartenance est visible dans de nombreux autres contextes ethnographiques où la question des migra-

\footnotetext{
4 Outre la vaste littérature sur les cimetières en tant qu'espaces de construction de la mémoire collective (cf. par exemple Ariès, 1989 ; Vovelle, 1983 ; Vincent-Thomas, 1975 et 1982) il existe aussi des textes qui traitent de la question du rôle des rites funéraires et des sépultures dans la construction de la communauté locale et de la relation entre la communauté des vivants et des morts, comme le livre classique de Douglass(1969) Death in Murelaga.
} 
tions et des diasporas est présente. Eric Gable, dans un article récent, The Funeral and Modernity in Manjaco (2006), consacré aux migrants Manjaks dans les villes de GuinéeBissau, soutient que les cérémonies funèbres sont l'occasion de démontrer sa réussite personnelle aux yeux des communautés rurales d'origine. Ces cérémonies sont utilisées comme des manières de légitimer des affirmations d'appartenance aux communautés locales dont on est issu. Au cours des funérailles, les Manjaks font appel à leur cosmopolitisme en tant que migrants et à leur urbanité, pour légitimer leur appartenance au lieu. En simplifiant, on pourrait dire qu'ils utilisent le cosmopolitisme visible dans leur succès et leur réussite comme un langage local d'affirmation d'appartenance et de prestige ; ils rendent local leur cosmopolitisme. Peter Geschiere (2005) quant à lui, dans un article consacré aux funérailles dans le sud Cameroun, met en doute le cosmopolitisme de ceux qui " vont ailleurs", mais qui investissent dans les funérailles pour affirmer leur appartenance à leurs communautés d'origine.

Les travaux de Françoise Lestage (2008) sur la migration mexicaine aux États-Unis et sur les processus de rapatriement des corps montrent comment on en est venu à créer une industrie de rapatriement dont diverses agences funéraires et des organes de l'État mexicain sont les principaux acteurs. De tels processus révèlent, une fois encore, la relation entre migrations et lieux, mais cette fois au niveau de la construction du nationalisme. Pour l'État mexicain, les migrants ont cessé d'être un poids ou un excédent pour devenir les principaux acteurs du développement, ce qui les autorise à posséder des droits et des privilèges que l'État ne leur accordait pas auparavant, telles la double nationalité et l'aide au retour des défunts.

Enfin, Engseng Ho (2006), dans sa recherche sur la diaspora yéménite dans I'Océan indien, montre comment les tombes sont des nœuds de circuits de populations qui relient la Péninsule arabique au sud de la Chine. Les tombes hadrami, éparpillées sur cette surface du globe, représentent des générations de Yéménites ayant exploré les routes commerciales de l'Océan Indien qui, en tant que tels, matérialisent la mémoire séculaire du déplacement vécu par les hadrami de l'époque coloniale à l'époque contemporaine. Ce sont les sépultures qui marquent les lieux d'appartenance d'une vaste diaspora ; elles ne renvoient pas seulement à un unique lieu d'origine, mais aux différents lieux qui la composent.

Ce qui ressort des exemples brièvement présentés ci-dessus, c'est précisément la dimension tellurique de la mort et son rôle dans des contextes migratoires et diasporiques très différents. En outre, la manière dont, dans tous ces contextes, on tente de maintenir une relation avec le lieu d'appartenance originel contient implicitement la notion de " bonne mort ", une notion essentielle dans l'œuvre de Bloch et Parry notamment. Le souci de maintenir les liens avec la terre d'origine est directement en relation avec l'idée que l'on ne réussit une " bonne mort " et une bonne relation entre le monde des vivants et le monde des morts que si ce lien est préservé.

Dans cet article, nous nous interrogerons sur les formes de la " bonne mort " chez les Guinéens et les Bangladais au Portugal. Pour répondre à cette question, nous commencerons par contextualiser les flux migratoires qui relient la Guinée-Bissau et le Bangladesh au Portugal puis nous aborderons les discours et les pratiques concernant la mort et les cérémonies funèbres dans les deux cas en question. Cet exercice comparatif permettra de discuter du transnationalisme migrant et des pratiques relatives à la mort et au mourir. 


\section{Les Guinéens et les Bangladais au Portugal}

Les Guinéens représentent aujourd'hui encore une des principales populations migrantes au Portugal à cause de leur importance démographique et de la durée des flux migratoires qui lient ces deux pays. La migration du Bangladesh est la plus récente et constitue une minorité représentative parmi les immigrés au Portugal. La situation de ces deux populations sur le marché du travail est bien distincte : les premiers sont associés aux secteurs les plus défavorisés du marché du travail, comme la construction civile et le travail domestique, tandis que les seconds ont misé sur les activités entrepreneuriales. Nous allons maintenant développer ces différences.

\section{Les Guinéens au Portugal}

Les Guinéens ont une longue tradition d'émigration. S'ils sont nombreux au Sénégal, en France et en Hollande (Machado, 2002), le Portugal s'est avéré très tôt une destination préférentielle, en raison de certaines affinités linguistiques et de liens avec des immigrés auparavant.

L'émigration au Portugal a commencé après la révolution du 25 avril 1974 et l'indépendance de la Guinée-Bissau, mais c'est surtout à partir de 1984, avec la fin du régime pro-soviétique de Luís Cabral et l'ouverture du pays à un modèle économique et social plus occidental, qu'elle va s'intensifier (Machado, 2002 ; Saraiva, 2008 ; Quintino, 2004). La situation de précarité et de pauvreté en Guinée-Bissau va s'aggraver avec la crise politique et le conflit armé de 1998, lesquels entraînent une augmentation du nombre de personnes fuyant leur pays et cherchant refuge au Portugal. En 2005, les données officielles comptabilisent 25148 Guinéens, mais leur nombre est sans aucun doute supérieur puisque beaucoup n'ont pas de statut légal.

Actuellement, une grande majorité des familles guinéennes a un ou plusieurs membres qui résident au Portugal et on observe un mouvement constant de personnes à l'aéroport de Lisbonne les jours de vol vers la Guinée ainsi que dans les rassemblements de Guinéens au centre de la ville (Rossio et Martim Moniz) ou lors du marché dominical où se font nombre d'échanges de biens (Saraiva, 2008 : 256).

La grande concentration des Guinéens continue d'être la zone de l'Aire métropolitaine de Lisbonne où la population résidente officielle comptait en 2010 environ 19187 personnes ; la Guinée fait ainsi partie des six pays dont le nombre total d'émigrants au Portugal est le plus important ${ }^{5}$.

La Guinée-Bissau est un petit pays d'environ 1,2 million d'individus, avec une grande diversité ethnique, où I'on compte près de vingt-trois groupes différents (Einarsdottir, 2000). On peut définir cette diversité en termes de filiation religieuse : les groupes de l'intérieur sont islamisés alors que ceux de la côte continuent d'observer des pratiques animistes, malgré la pénétration d'autres religions, en particulier évangélistes, ces vingt dernières années. Mais la filiation religieuse ne suit pas toujours une stricte division de groupe (Jao, 1995) et il existe de nombreuses combinaisons entre des traditions religieuses animistes et le christianisme, notamment le catholicisme et le protestantisme.

5 Données de I'Observatoire de I'Immigration (2012) Relatório de Imigração, Fronteiras e Asilo (RIFA). 
Au Portugal, pratiquement tous les groupes ethniques sont représentés, même si certains sont plus nombreux que d'autres. Malgré cette diversité dans la diaspora, se manifeste la revendication d'une origine commune. D'une manière générale, I'origine nationale l'emporte sur les divisions ethniques (Bordonaro et Pussetti, 2006 : 133). La ville de Bissau, la capitale, surgit comme un symbole central où se rassemblent toutes les différences ethniques, religieuses et générationnelles. Elle est aussi un point de passage obligatoire pour le départ (Saraiva, $2008: 257)$.

Au Portugal les Guinéens recréent leur identité en réélaborant les références et les codes du pays d'origine en relation avec les codes du pays de destination. Les divers éléments qui forment un "bloc ethnique " - comme le territoire, la langue, la couleur de peau, les codes vestimentaires, l'alimentation, la musique, la danse - sont constamment négociés et manipulés par chacun de manière à construire son identité en relation avec les autres Guinéens et les Portugais (Quintino, 2004 ; Saraiva, 2008).

\section{Les Bangladais au Portugal}

Les migrants originaires du Bangladesh sont arrivés au Portugal à partir de 1986, mais c'est entre 1995 et 2003 que cette population a augmenté de manière significative : le nombre de personnes légalement enregistrées dans les services de l'immigration - en plus des 4000 enregistrés au consulat général du Bangladesh à Porto - est passé de quarante-sept à $2243^{6}$. Début 2009, le nombre de citoyens du Bangladesh enregistrés au consulat dépassait déjà 4500 personnes.

Comme en Espagne et en Italie, la majorité de ces migrants est originaire de couches sociales intermédiaires, définies au Bangladesh comme la nouvelle et influente " classe moyenne ", urbanisée et instruite. Pour cette population, venir en Europe n'est pas une manière d'échapper à la pauvreté, mais bien plutôt une stratégie pour accéder à ce que I'on appelle au Bangladesh I'adhunik - le " moderne " - et, en même temps, au statut d'adulte (Mapril, 2007).

Les chaînes migratoires qui ont servi de support à la formation de ce flux sont directement liées aux processus de régularisation mis en place dans différents pays de l'Europe méridionale, depuis la fin des années 1980 jusqu'à aujourd'hui. Beaucoup de Bangladais se trouvaient déjà en Europe et ne sont arrivés au Portugal que pour rechercher des opportunités de légalisation impossibles à obtenir dans d'autres contextes. La majorité d'entre eux a suivi ceux qui se sont établis en Allemagne, France, Italie et Espagne au cours des dernières décennies (Knights, 1996 et 1997). Par ailleurs, la modification de la situation sociale et économique des pays de l'Europe méridionale, du fait de leur appartenance à l'espace communautaire, a modifié leur position face à la division internationale du travail (cf. Malheiros, 1996 ; Baganha et al., 1999 ; King et al., 2000). Ces changements ont contribué à ralentir le rythme des migrations intra européennes et ont entraîné l'arrivée de nouveaux immigrants non originaires des anciens espaces coloniaux. Face à ces " nouveaux " flux migratoires, beaucoup de ces pays ont développé des législations et des programmes spécifiques en vue de la régularisation des immigrants. À l'image du cas italien, de nombreux Bangladais se sont déplacés jusqu'au Portugal dans le cadre de processus de régularisation développés par les autorités portugaises en 1992, 1996 et 2001-2004 et/ou pour rejoindre leurs amis et les membres de leurs familles.

6 Ces données ont été calculées en additionnant le nombre de Bangladais ayant un statut de résident au Portugal et celui des Bangladais ayant un statut de permanent entre 2001 et les dernières données statistiques portant sur l'année 2003. 
Bien que beaucoup soient arrivés seuls, quand nous avons commencé notre d'enquête nous observions cependant la présence d'enfants et de femmes, ce qui indiquait que certains commençaient à procéder au regroupement familial. Cela dit, il faut comprendre que cette population est segmentée en fonction du moment de sa migration. Ainsi, d'un côté, on a les pionniers - arrivés à la fin des années 1980, début des années 1990 - qui ont commencé à travailler dans les secteurs les plus défavorisés et qui, aujourd'hui, sont propriétaires de différents commerces. Ils ont constitué leurs unités domestiques et sont des exemples de succès et de réussite. D'un autre côté, on a les nouveaux venus, ceux qu'on appelle les bachelors ou freshies - ils ont migré dans le cadre des processus de régularisation de 2001 - qui continuent encore aujourd'hui d'arriver. Ils travaillent dans les secteurs les plus précaires ou sur le marché du travail créé par les pionniers et dans la majorité des cas sont célibataires ; seuls à Lisbonne et souvent sans famille au Portugal, leur projet migratoire est encore incertain.

Dans ces contextes migratoires, comment sont gérés la mort et le fait de mourir?

\section{La " valise " du défunt : le cas des Guinéens}

Dans son livre sur l'immigration guinéenne au Portugal, Celeste Quintino (2004: 35) parle d'une "trans-identité ", forgée dans la relation constante entre les immigrants et la société d'accueil, ainsi qu'avec la communauté d'origine et sous-tendue par le désir de retourner un jour en Guinée. Le désir de retour peut apparaître contradictoire avec l'idée d'un " paradis " portugais. II est aussi symptomatique du dépaysement et du sentiment de non-intégration dont souffrent les Guinéens.

La mort est précisément une des situations qui stimule le désir de retour au pays et le renouvellement des liens - réels et symboliques - avec les communautés d'origine. Traversant les différences de statut socio-économique et les alliances ethniques, le tchon (lieu/pays d'origine) fonctionne comme référence territoriale et ethnique cruciale. Sa construction symbolique se fonde sur la langue et un passé commun, enracinée dans l'organisation sociale de la tabanka (village) et dans les devoirs de chacun envers son lignage et sa moransa (famille étendue vivant dans le même espace physique). Ainsi se construit un " ethnicity package " négocié en permanence et manipulé dans un processus symbolique constant de liens avec les origines.

Les Guinéens au Portugal ont un mouvement associatif fort : il existe plus de cinquante associations, dont la plupart sont des ONG. Dans leur grande majorité, leurs objectifs visent l'appui aux immigrants : leurs relations avec l'administration et les institutions portugaises, l'obtention du travail, la légalisation de statut, etc., mais aussi la promotion de la solidarité entre Guinéens, l'aide aux régions d'origine en Guinée (par exemple, par la construction ou la gestion d'équipements publics), les programmes de coopération, etc. Beaucoup d'entre elles sont constituées sur la base d'affinités régionales et locales, regroupant initialement les personnes provenant d'une région déterminée, mais elles finissent par compter parmi leurs membres des personnes originaires de diverses régions de la Guinée.

De plus, elles s'occupent de l'organisation des rites de passage et des fêtes cycliques ou annuelles, de caractère profane ou religieux, où se conjuguent des pratiques catholiques, musulmanes et animistes. Dans ce cadre, une des fonctions primordiales de ces associations est de fournir le support économique permettant le retour au pays des cadavres ou, du moins, la venue de la famille, ou encore l'envoi des biens du défunt en Guinée. II s'agit de préserver la relation avec les lieux d'origine, condition sine qua non pour que l'idéal de la bonne mort se concrétise. 
Voyons comment cela se passe dans la pratique.

Plusieurs associations guinéennes demandent à leurs membres le paiement d'une cotisation qui alimente un fonds commun. II sert à payer le rapatriement des corps, lorsque la famille le décide. Dans bien des cas, cela s'avère impossible. Aussi ce qui se pratique, c'est un rapatriement, physique et symbolique, des divers éléments qui relient le défunt et sa famille migrante au territoire et à la famille d'origine en Guinée-Bissau. L'envoi de la valise et la réalisation du toka chur en Guinée sont les deux éléments les plus significatifs de ce processus.

Les sources ethnographiques relatives à ces pratiques proviennent d'une des associations les plus actives en matière de prestations autour de la mort et de données recueillies lors de séjours en Guinée. Cette association, fondée en 1992, est l'une des plus engagées à maintenir les liens avec le lieu d'origine grâce aux moyens économiques que permet le paiement d'une cotisation bisannuelle de ses membres. II s'agit d'une association d'origine Manjak, un groupe à forte tradition migratoire (de nombreux Manjaks vivent en France, où il existe une association de compatriotes, qui travaille en collaboration avec I'association portugaise).

L'association est fortement impliquée dans l'aide à ses membres en cas de maladie et de mort. En cas de mort, tout membre a droit à une aide économique en vue de l'accomplissement des rituels. Dans la grande majorité des cas (environ $90 \%$ ), les défunts sont enterrés dans des cimetières de Lisbonne et l'association paie les funérailles ${ }^{7}$. Si le corps est envoyé en Guinée, I'association donne le même montant, et la dépense restante (qui avoisine 5000 euros) devra être supportée par la famille. Mais il est bien souvent difficile à une famille guinéenne de supporter de telles dépenses et, dans ce cas, le plus important est la ritualisation de la relation avec le lieu d'origine et la famille en Guinée, de manière à atteindre l'idéal de la " bonne mort ". Cette relation se concrétise en termes symboliques à travers diverses actions et représentations rituelles qui établissent un pont physique ou symbolique avec le lieu d'origine. L'une d'elles, qui dépasse les divergences ethniques et/ou religieuses, puisqu'elle est utilisée par des catholiques, des évangélistes, des musulmans et des animistes, est le rituel de la valise du mort. Après l'enterrement, c'est la famille proche qui s'en charge. Cette valise est achetée si le défunt n'en possédait pas, elle est remplie de ses biens, surtout de vêtements, de bijoux, de divers morceaux de tissus, de mouchoirs, de serviettes, de couvertures et de tissus penti (tissus fabriqués manuellement sur des métiers à tisser, particulièrement valorisés en Guinée et un peu dans toute l'Afrique occidentale) (Saraiva, 2008).

En plus de la participation aux frais de l'enterrement, l'association octroie 250 euros par défunt, montant qui pourra être complété par la famille en diaspora si celle-ci en a les moyens et qui est, théoriquement, placé " au fond de la valise ". Le transport doit obligatoirement être accompagné, la valise ne voyage pas seule. Portée par un proche, elle est remise in loco à la famille du mort. Comme nous le dira un de nos informateurs : "C'est la famille là-bas qui décide quoi faire des biens et comment les distribuer, c'est la famille qui doit recevoir la valise. Et tous ici ont de la famille en Guinée ». Ces propos synthétisent deux idées fondamentales. En premier lieu, le renouvellement de la continuité de la famille au-delà des frontières et des mobilités de ses membres se fait et les devoirs échoient à la " famille directe ", qui est aussi la plus à même de recevoir les bienfaits d'un défunt qui accède au statut d'ancêtre protecteur, mais qui peut aussi subir la vengeance d'un esprit pour lequel n'ont pas été intégralement accomplis tous les rituels. En deuxième lieu, c'est

7 Une dépense tournant, en 2011, autour de 1000 à 1400 euros. 
justement lorsque les mobilités physiques cessent, par force que, dans l'impossibilité de faire rentrer le défunt lui-même, ce qui symbolise l'individu, ses biens, doit retourner au lieu d'où il est parti initialement, et ainsi refermer le cycle de liaison avec le tchon.

Les biens de la valise appartenaient au défunt quand il était en vie ou proviennent des offrandes réalisées par des membres de la famille et des amis à l'occasion de sa mort. Personne ne se rend à un enterrement sans apporter un don : du linge, des morceaux de tissu, des boissons et des aliments. Le linge et le tissu sont distribués ou sont mis dans la valise du défunt. On dit que le défunt a besoin de ses affaires, de son linge et du tissu pour sa vie dans l'autre monde : "le mort n'emporte dans l'autre monde que ce qu'on lui offre ; ce qui était à lui vivant est donné à la famille, celle d'ici et celle de là-bas ; ses propres affaires ne vont pas dans l'autre monde, seulement ce qui a été offert y va ".

La logique du caractère obligatoire des offrandes n'est pas strictement altruiste : tout ce que les vivants font pour les morts leur reviendra puisque ce sont des dons qui vont enrichir l'autre monde dont ils feront tous partie un jour : "Ce que moi, encore vivant, j'offre à quelqu'un, même si cette personne est encore vivante, est/sera à moi dans l'autre monde ; quand on offre à un mort, ce que l'on offre est pour lui dans l'autre monde ".

Malgré leur apparente contradiction, ces deux considérations sont complémentaires : ce qui était au mort part dans la famille réelle, en diaspora et dans le lieu d'origine ; et tout ce qui est offert par ceux qui viennent rendre les derniers hommages à l'enterrement est symboliquement offert à l'esprit du mort et au monde des esprits en général. Autrement dit, la générosité de ceux qui rendent leur dernier hommage au mort leur revient. En effet, en garantissant au " nouveau-défunt " une valise pleine, ils expriment en même temps leur reconnaissance envers leurs ancêtres qui vivent dans I'autre monde. Ainsi, ces derniers pourront recevoir des dons de l'esprit qui symboliquement les emmène jusque dans cet autre monde et qui redistribue leur surplus, comme cela est explicité par le fondateur de l'association: "On doit offrir le plus possible; si l'esprit arrive dans le monde des morts avec beaucoup de linge et de tissus, il offre aux autres".

L'organisation de la valise débute normalement avec la cérémonie qui a lieu après I'enterrement où tous se réunissent, idéalement dans la maison du défunt, sinon, dans un espace loué ou emprunté, plus grand s'il s'agit d'un enterrement très prisé, et commun aux animistes, aux catholiques et aux musulmans. D'après la tradition orale, au cours de cette réunion on doit sacrifier un ou plusieurs animaux - au Portugal, une chèvre ou un chevreau - qui doivent ensuite être cuisinés et partagés lors d'un repas communautaire. Depuis environ dix ans, et en raison des lois imposées par l'Union européenne et par l'État portugais, ces animaux sont en règle générale achetés une fois abattus ${ }^{8}$ pour être cuisinés et consommés collectivement.

Tout au long de ces heures passées ensemble, on chante ou on prie pour le défunt, les gens arrivent les dons, mais aussi avec des boissons (vin, eau-de-vie, porto, whisky, jus de fruits et sodas) que I'on utilise pour le darmar. II s'agit de verser par terre un peu du liquide pour que les esprits des ancêtres puissent boire eux aussi. Ces derniers sont toujours aux côtés des vivants, surtout à un moment où I'on célèbre l'entrée d'un nouvel esprit. Les ancêtres habitent un monde où la mobilité est sans limites. Ils sont présents dans les cérémonies, en Guinée comme au Portugal, et dans la diaspora : "Ils n'ont pas besoin de prendre l'avion, comme nous, les vivants. S'il y a une cérémonie, ils viennent, ils sont ici à nos côtés, et il faut leur donner à boire et à manger ".

8 Les musulmans jouissent d'espaces spécifiques pour effectuer le sacrifice d'animaux. 
La relation entre deux espaces et entre les communautés liés par la mobilité des migrants et par la mobilité des esprits des ancêtres se concrétise dans la réciprocité des devoirs entre ceux qui " sont ici " et ceux qui " sont là " : on organise en Guinée les cérémonies pour quelqu'un qui meurt et est enterré au Portugal, et vice-versa au Portugal, pour quelqu'un qui meurt et est enterré en Guinée.

Manger, boire, offrir des tissus et le sang des animaux sacrifiés, voilà des offrandes des vivants aux morts présentes dans la majorité des cérémonies en Guinée et au Portugal et notamment pour les rituels funéraires qui établissent un pont entre les deux mondes.

Pour comprendre ce rituel complexe, il convient de renvoyer à ce que Quintino (2004) appelle I' " ethnic package " des Guinéens au Portugal et à ce qui les unit en diaspora et traverse donc les différents groupes ethniques : la commensalité, I'habillement, les tissus et la croyance en une présence permanente des esprits ancestraux dans la vie des vivants, ou, plutôt, l'annulation d'une séparation entre le monde des vivants et le monde des morts.

En réalité, toutes les familles en Guinée ont de la famille au Portugal, et elles sont dans I'attente (morale et réelle) d'une aide des migrants. II n'est pas concevable que ceux-ci y échappent ; le fait qu'une personne ait migré est synonyme de réussite et signifie qu'elle sera toujours dans une position économique où elle ne peut passer outre au devoir d'aider les membres de la famille restés en Guinée. Mais plus encore, les funérailles sont des événements d'une haute valeur sociale, où l'on peut exhiber cette réussite (cf. Gable, 2006). C'est aussi pour cela que ce sont des émigrants ayant réussi dont le corps peut être rapatrié, alors que les frais sont élevés. S'il y a ici une logique de réussite et de triomphe économique, les personnes âgées ayant atteint des positions sociales élevées au sein de la communauté des immigrants guinéens au Portugal savent elles aussi que leurs corps retourneront pour être enterrés dans le tchon d'origine : "Si je meurs, mes enfants m'ont déjà dit qu'ils m'emmèneront en Guinée [...]. Un omi garandi a sa maison organisée là-bas en Guinée, il a déjà noté l'endroit où il veut être enterré, pour cela, s'il vient au Portugal et meurt, son corps sera envoyé là-bas ".

En dehors des considérations économiques, la différence de traitement entre vieux et jeunes, met en évidence non seulement la révérence à l'égard des plus vieux, mais aussi une hégémonie réelle et symbolique exercée par les anciens envers les plus jeunes. Cette gérontocratie que Ramon Sarró détecte également parmi les Baga de Guinée Conakry (Sarró, 2009) semble prévaloir dans la migration et dans la manière dont la réussite dans I'aventure migratoire a conféré du prestige.

Dans une logique migratoire de recherche de la réussite (économique, sociale), un jeune $^{9}$ est quelqu'un qui n'a pas encore atteint cet objectif ni le prestige qui en découle. Or cette hégémonie des vieux sur les jeunes se poursuit au-delà de la mort et c'est cela qui fait sens. Pour Kopytoff (1971), un vieil homme est déjà considéré comme un ancêtre potentiel. Sa mort est une occasion de fête, de célébration de son entrée dans le monde des défunts et de son ascension vers le statut d'ancêtre, potentiellement protecteur de son lignage. Une personne âgée possède du prestige et du pouvoir (économique, social, religieux), qu'il transporte dans le monde de l'au-delà. La mort d'un omi garandi (personne âgée) doit alors être fêtée comme il se doit, dans son lieu d'origine et avec les siens - sa famille élargie, son lignage et les habitants de sa moransa -, afin d'assurer la

9 Depuis 1992, treize jeunes sont morts et enterrés au Portugal. Les funérailles ont été assurées par I'association et pour tous on a envoyé la valise. 
fidélisation de cet ancêtre. II doit être l'objet de beaucoup plus d'offrandes et les cérémonies devront être beaucoup plus somptueuses que celles d'un jeune.

Cette logique est la même que celle des offrandes du chur et du tokachur des religions animistes, telles qu'elles sont pratiquées par les Papel, les Manjaks, les Mancagnes, les Bijagós et les Balantas, ainsi que par des individus qui se considèrent comme catholiques, mais pratiquant également les cérémonies d' " uso " (" tradition " en créole). Dans le cas des Papel, les premières cérémonies funèbres incluent l'enveloppement du défunt pendant plusieurs jours, leur nombre variant selon la position sociale, religieuse et lignagère de la personne ( $d$ 'autant plus de jours que la position est élevée). Le corps est enveloppé dans des pièces de tissus offertes à la famille en deuil et au défunt par tous les participants aux funérailles. Ces tissus sont ainsi symboliquement emportés par l'esprit du défunt jusque dans l'autre monde où ils deviendront ses propres biens. Un défunt âgé et prestigieux doit en emporter beaucoup. Ils seront redistribués aux ancêtres de tous ceux qui ont apporté des offrandes aux funérailles. Lors de cette cérémonie des disputes éclatent, car tous veulent que leurs morceaux de tissu soient placés sur le corps du défunt, ce qui n'est pas toujours le cas. La garantie que le tissu que l'on offre arrive dans l'autre monde est en même temps la garantie que les ancêtres seront heureux et maintiendront une bonne relation avec leurs descendants.

Dans les cérémonies de secondes funérailles (toka chur), c'est le sang des animaux sacrifiés qui est offert aux ancêtres (Saraiva, 2003, 2004, 2004b et 2008). Mais le toka chur n'a vraiment une signification importante que pour la mort d'une personne âgée, qui a accompli toutes les étapes de sa vie et réussi à atteindre la plénitude, et lorsqu'on estime qu'il fait une entrée somptueuse dans le monde de l'au-delà pour devenir un ancêtre. Un toka chur d'une personne âgée, c'est un festival de danses, de chants, de commensalité élargie et de sacrifices d'animaux qui se prolonge durant plusieurs jours ; celui d'un enfant ou d'un jeune est une cérémonie brève et simple, pleine de tristesse.

Dans le cas de groupes cités plus haut, la réalisation du toka chur est essentielle pour que l'esprit du défunt parvienne à sa place dans le monde des morts, et que soit ainsi obtenue une belle mort (Saraiva, 2008) ${ }^{10}$. Par exemple, pour les Papel, le toka chur doit obligatoirement avoir lieu au tchon Papel; un toka chur fait au Portugal n'a aucune valeur. Cela nous ramène aux considérations sur l'hégémonie des vieux sur les jeunes. On considère que ces derniers doivent travailler et réussir, dès lors que l'une de leurs contributions consistera à s'occuper et à financer le toka chur des vieux de la famille proche : " Je fais des heures supplémentaires dans mon travail pour pouvoir avoir de l'argent pour aller en Guinée faire le toka chur de mon père. Les vaches et le reste des animaux sont chers... en plus du prix du billet d'avion ".

II est impossible au Portugal d'enterrer un corps enveloppé dans des centaines de morceaux de tissu et en l'absence de cercueil, les morceaux de tissu sont placés dans la tombe. On calcule alors le montant et la valeur des tissus, adaptés au contexte ${ }^{11}$. Par

\footnotetext{
10 Les secondes cérémonies d'obsèques devraient avoir lieu théoriquement tout de suite après les funérailles. Dans la pratique, ce n'est presque jamais le cas et en général elles ont lieu des mois ou des années plus tard. On peut se référer à d'autres contextes ethnographiques où la distance temporelle des deux cérémonies correspond à une période faisant coïncider la décomposition et la séparation de la chair et des os, le voyage de l'esprit jusqu'au monde des morts et la période de deuil, comme c'est le cas chez les Berawan de Bornéo, étudiés par Metcalf (1985).

11 L'importance des tissus est attestée par les agences funéraires qui constatent souvent, dans les transferts de défunts, que leur poids est de beaucoup supérieur à celui du corps, surtout si ce sont des tissus penti, particulièrement lourds. Cela augmente d'autant le prix du transport du cercueil.
} 
I'intermédiaire d'un artifice d'adaptabilité symbolique on contourne les règles et les contingences de la condition d'immigrant : on dit par exemple qu'un tissu correspond à $\mathrm{X}$ ou Y tissus en Guinée.

On a ainsi un réseau transnational où esprits, personnes, cadavres et biens circulent dans deux ensembles de mondes parallèles : les mondes physiques, concrets, des deux contextes nationaux - celui du pays d'origine, la Guinée, et celui du pays d'accueil, le Portugal - et les deux univers qui existent au-delà de toute frontière ou de distances spatiales, le monde des vivants et celui des morts (Saraiva, 2008 : 267).

\section{" Ici personne ne prie pour toi ": le cas des Bangladais}

Dans le cas des Bangladais, on observe une logique comparable à celle que nous venons de décrire pour les Guinéens, mais elle se manifeste sous des formes différentes. La gestion de la mort et de l'enterrement des migrants est directement liée à la réciprocité et la responsabilité morale qui lient des parents entre le Portugal et le Bangladesh. Le projet migratoire implique l'obligation d'envoyer de l'argent aux membres de la famille dans la desh (terme bengali pour désigner le Bangladesh), obligation qui est associée à la nature corporative de l'investissement migratoire - auquel participent fréquemment divers membres de l'unité domestique du migrant. Au moment de la mort du probashi, le retour à l'origine symbolise cette relation pérenne qui n'est pas seulement tellurique, mais aussi de parenté. Comment se présente cette relation?

Depuis 1986, date d'arrivée du premier Bangladais, quatorze bengalis sont morts dans différentes circonstances (maladies prolongées, accidents de travail et crimes présumés) et tous ont été enterrés au Bangladesh, sauf un, sur lequel nous reviendrons plus loin ${ }^{12}$.

Les dépenses et l'organisation du transfert du corps sont à la charge des pionniers. En tant que symboles de succès et de réussite, ils assument fréquemment le rôle de leaders de la " communauté " et c'est précisément en tant que badralok (désignation bengali pour dire " homme important ") qu'ils sont appelés pour résoudre des situations de conflit ou pour gérer la mort d'un compatriote. Ce sont eux qui, aidés d'autres personnes, préparent les cérémonies funèbres en collaboration avec les principales institutions islamiques au Portugal.

II convient de rappeler ici que, en 2006, les musulmans bengali ont créé au centre de la ville de Lisbonne, la mosquée Baitul Mukarram (nom de la mosquée centrale de Dhaka, la capitale du Bangladesh). Elle peut accueillir plus de cinq cents personnes et elle est autonome par rapport à d'autres institutions islamiques portugaises dans le domaine cérémoniel (Mapril, 2005). Néanmoins, elle ne dispose pas d'installations permettant d'organiser des cérémonies funèbres et, en conséquence, les corps sont toujours acheminés

12 Une grave maladie ou la mort d'un parent au Bangladesh peut entraîner un voyage. Cependant, pour des raisons financières ou de statut légal, le déplacement n'est pas toujours possible d'autant que le défunt doit être enterré le plus rapidement possible. Une des solutions est de réaliser certaines cérémonies à Lisbonne en même temps qu'elles ont lieu au Bangladesh. Une autre manière de gérer la mort à distance consiste à demander à des amis et à des membres de la famille de réaliser des du'as (des prières) au nom des défunts. 
vers la Mosquée Centrale de Lisbonne ou la Mosquée Hazrat Bilal, à Porto ${ }^{13}$.

C'est au sein de ces institutions que se fait partiellement la gestion des rituels funéraires chez les Bangladais. Ce sont les fonctionnaires de ces mosquées qui pratiquent les lavages (ghosul), les ablutions (wusu) et enveloppent le cadavre dans le kafan, le linceul blanc, également porté par les pèlerins pendant le Hajj. Il s'agit là d'une tâche effectuée par des hommes ou par des femmes selon le sexe du défunt. Ensuite, le corps est embaumé, comme le prescrit la loi pour les transferts des défunts, et mis dans un cercueil scellé pour le retour au Bangladesh. Avant de sceller le cercueil, on transporte le corps dans la salle de prières ou, dans le cas de la mosquée centrale de Lisbonne, dans le patio intérieur où I'on récite la salat-ul-janazah, l'oraison funèbre. D'après nos interlocuteurs, pour cette oraison les croyants doivent être en nombre significatif dans la mesure où plus la congrégation est grande, plus le mérite, le sowab, que " recevra " le défunt sera grand. Ainsi n'est-il pas inhabituel que de nombreux Bangladais se déplacent de tout le pays pour assister à ces prières, parfois même littéralement " recrutés " pour y participer, que ces personnes aient un lien avec le défunt ou pas. Selon certains de mes interlocuteurs, c'est le devoir de tous de participer, car tous auront un jour besoin de l'engagement et de l'assistance des autres. Cette congrégation est exclusivement masculine. L'épouse du défunt, qu'elle se trouve au Portugal ou au Bangladesh, doit rester à la maison, en contact minimum avec l'extérieur pendant une longue période (jusqu'à quarante jours). Elle reçoit la visite d'autres femmes, mais il est souvent mentionné qu'elle doit rester éloignée des hommes n'appartenant pas au lignage patrilinéaire (Gardner, 1998).

Les jours suivants, au cours des prières quotidiennes dans la mosquée Baitul Mukarram, on rappelle la mémoire du défunt et on demande à la congrégation de prier pour lui. Le rapatriement du corps se fait dès que possible par une agence funéraire. Le prix du transport est équivalent à la moitié d'un billet de tourisme qu'il faut régler à l'agence. II faut aussi demander les documents adéquats au Consulat Honoraire du Bangladesh. Toutes ces dépenses sont assurées par des collectes organisées parmi les pionniers et au sein d'associations régionales informelles. Pour les pionniers, en tant que membres ayant bien réussi, ces dons d'argent représentent l'obligation de redistribuer leur richesse dans la " communauté ". Ils sont aussi vus comme de bonnes actions (waqf) qui leur permettront d'avoir du mérite, capital sacré (voir infra). Simultanément, ils sont une manière de légitimer leur autorité comme personnes importantes dans la communauté qui dépend d'elles. Les dix-huit associations régionales informelles qui participent aux collectes d'argent se sont constituées ces dernières années. Elles reproduisent les diverses appartenances des migrants et se consacrent au bien-être de leurs membres en utilisant notamment les bénéfices du capital accumulé. II est utilisé non seulement pour des investissements commerciaux, mais aussi en cas de nécessité ou lors du décès d'un membre. Dans ces dernières circonstances, il revient à ceux qui restent, indépendamment de leur position dans la migration, d'aider à financer le rapatriement de leur compatriote. II n'y a pas de montant fixé, mais les contributions des principales figures de la " communauté " se situent dans la plus haute tranche. Les collectes sont aussi destinées à la famille ou à la veuve restée au Bangladesh ${ }^{14}$.

\footnotetext{
13 La Mosquée Centrale de Lisbonne existait déjà lors de l'arrivée des Bangladais. Ce lieu de culte a été inauguré en 1985, alors que les mosquées de Laranjeiro et d'Odivelas développaient déjà leurs activités respectivement depuis 1982 et 1983. Il est lié aux Indiens originaires du Mozambique et à la Communauté Islamique de Lisbonne (C.I.L.) fondée en 1968 (Tiesler, 2000 ; Vakil, 2003). La mosquée Hazrat Bilal inaugurée en 2001, après treize ans de négociations avec les autorités, est un des espaces de culte fréquentés par beaucoup des Bangladais résidant dans cette ville.

$14 \mathrm{Ce}$ montant a atteint 5000 euros pour un cas connu.
} 
Il revient à la famille d'aller chercher le corps à l'aéroport. Dans certains cas on loue une ambulance qui transporte la dépouille jusqu'au cimetière où on organise l'enterrement. Le cercueil est ouvert pour montrer le corps puis enterré dans le desh, le visage tourné vers la Mecque. On peut aussi enterrer le corps sur les terres familiales, à côté d'autres parents, ou bien tout près du mazaar des fameux pirs, là où ont été enterrés des saints célèbres (Gardner, 1998 et 2002). Au nord-est du Bangladesh, où l'importance historique du " pirisme " est structurante et permet de comprendre les dynamiques d'islamisation du golfe du Bengale (Eaton, 1993), une telle pratique est considérée comme de très bon augure pour le défunt, puisque celui-ci est protégé par le charisme de l'homme saint.

Mais pourquoi ne pas enterrer le défunt au Portugal comme des membres de la communauté islamique de Lisbonne le demandent puisqu'il existe plusieurs carrés islamiques $^{15}$ ? Parce qu'envoyer les corps des défunts dans le pays d'origine est considéré comme une pratique non islamique, dans la mesure où le corps doit être enterré le plus rapidement possible, ce qui n'autorise ni embaumement ni transferts. Une telle critique est aussi exprimée par certains de nos interlocuteurs, en particulier ceux qui ont eu une expérience migratoire sur plusieurs générations et pour qui l'enterrement doit être effectué rapidement, là où se trouve la personne. Le transfert au Bangladesh représente non seulement une dépense inutile, mais aussi une démarche ne tenant pas compte des orientations doctrinaires. Mais pour d'autres personnes, la préoccupation d'enterrer les morts au Bangladesh relève d'une croyance généralisée en l'obligation de "retourner " à la desh, près de ses parents (atyio). Pour Ali par exemple : "Ici, personne ne prie pour toi parce que personne ne te connaît. Au Bangladesh, quand les personnes passent dans le cimetière, elles se souviennent de toi et par conséquent font un du'a en ton nom ". Pour lui, chaque prière faite au nom du défunt jouera en sa faveur dans le futur, quand ses comportements dans la vie seront estimés par Dieu. C'est pourquoi il est nécessaire d'enterrer les personnes près de leurs proches. Et lorsque le jugement dernier arrivera, tous - à commencer par les morts - devront rendre des comptes sur leurs actions sur terre. Avoir des parents qui font des prières ou qui tiennent des assemblées de dévotion au nom du ou en hommage au défunt est donc une manière d'acquérir du mérite.

Cette idée va de pair avec les conceptions de la mort partagées par nombre de nos interlocuteurs qui présentent des similitudes avec ce qui a été décrit dans différents contextes au Bangladesh (Kotalová, 1994 ; Gardner, 1995). Ce qui se fait sur terre a des conséquences non seulement sur la vie, mais aussi après la mort. Les actions quotidiennes sont souvent jugées en termes moraux et selon les conséquences qu'elles peuvent avoir sur la vie après la mort. Ainsi certains actes que I'on accomplit permettent d'acquérir du mérite (sowab) et un capital sacré, indispensable pour entrer au paradis (janna) et échapper aux tourments de l'enfer (jahannan). La charité (lilla), sous forme de donations et d'offrandes ainsi que les bonnes actions (waqf), et le sacrifice de biens personnels en faveur de la " communauté ", sont des exemples de pratiques d'accumulation du mérite. De plus, dans le calendrier religieux, il existe des occasions de cumul " extraordinaire " de mérite, comme la nuit de la révélation (Shab-e-Qadr). Les prières effectuées la nuit du Shab-e-Qadr sont supposées " meilleures" que celles faites dans d'autres circonstances. Elles comportent plus de sowab que mille prières " ordinaires " et sont une occasion d'accumuler du capital sacré. Mais, après la mort, l'acquisition et l'accumulation de sowab ne sont possibles que grâce aux prières, aux supplications et aux réunions de dévotion au nom du défunt, faites par des membres de la famille et des amis qui contribuent à l'augmentation du capital sacré. D'où là la nécessité d'être enterré tout près des membres de la famille, des amis et des connaissances qui entretiennent le souvenir.

15 Actuellement il existe trois carrés islamiques qui se trouvent à Lumiar, à Odivelas et à Feijó. 
La relation entre l'enterrement au Bangladesh et le mérite (sowab) a été interprétée un peu différemment par Gardner (1993). Elle a montré comment pour de nombreux Bangladais, le terme bidesh (le pays étranger) renvoie à l'idée de terre d'abondance, de richesses et de réussites et celui de desh (village et région d'origine) est associé à la pauvreté et au manque d'opportunités. Le revers de la médaille est que bidesh est envisagé comme peu religieux et moralement menaçant face à un Bangladesh de dévotion et de valeurs. Puisque la mort n'est pas une fin, mais au contraire un autre chemin conduisant à Dieu, lequel dépend de pratiques religieuses pratiquées par les autres, mieux vaut alors être enterré dans un pays de personnes dévotes plutôt que dans un pays où existe un déficit de valeurs et de religion et où peu de musulmans veillent sur le mort. Être enterré au Portugal est donc bien souvent mis en relation avec une idée d'abandon.

Envoyer le corps au Bangladesh est une manière de se conformer à un idéal de " bonne mort " (Bloch et Parry, 1982). Dans les travaux ethnographiques consacrés au Bangladesh (Kotalová, 1994 ; Gardner, 2002 ; Garbin, 2004), la " bonne mort " est celle qui survient auprès des "siens ", de ses parents, des membres de l'unité domestique et de la lignée patrilinéaire et non pas seul ou entouré d'inconnus. L'idéal est de mourir chez soi, mais pour les migrants qui continuent de considérer que leur maison se trouve au Bangladesh, l'idéal est d'être rapatrié.

On retrouve cette même idée chez les musulmans bengalis de Londres où, comme le montre Gardner (1998), nombreux sont ceux qui ressentent la nécessité de transférer les défunts au Bangladesh, à Sylhet, pour que les parents voient le cadavre une dernière fois. Au Royaume-Uni, 60 à $70 \%$ des corps sont rapatriés (Gardner, 1998).

Cette préoccupation est telle que l'État bangladais a pris des mesures pour le rapatriement des corps des travailleurs expatriés. Dans le cadre de la politique d'émigration instaurée depuis le milieu des années 1980, l'État a mis en place en 1990 le Wage Earners Welfare Fund. Ce fonds visait à développer des programmes d'aide sociale destinés aux travailleurs migrants et à leurs familles. Ils incluaient le transfert des corps des migrants décédés ainsi qu'un soutien financier pour les familles. Les montants mis à disposition s'élèvent à près de 20000 takas. Depuis sa création, ce fonds a déjà permis de rapatrier les corps de 5000 travailleurs migrants (Siddiqui, 2001).

Selon des responsables, le rapatriement des probashis (émigrants en bengali) est une manière de rétribuer les sacrifices que beaucoup font pour le développement du pays. Les remises d'une partie des 5 millions environ de probashis employés au Royaume-Uni, en Arabie Saoudite, aux États-Unis, dans les Émirats, en Malaisie, en Italie, etc., sont considérées comme indispensables à l'économie et à la " modernisation " du pays. De sorte que financer le rapatriement des défunts est perçu comme un juste retour pour qui se " sacrifie " au nom du Bangladesh.

Qu'arrive-t-il quand les réseaux sociaux se délitent ou quand les parents meurent ou vivent tous dans le pays d'immigration?

Prenons le cas d'Alam, résidant au Portugal et où il faisait le circuit des foires depuis 1996. En 2001, il a un accident de voiture et se blesse gravement. Le projet était de le transporter à Dhaka, mais on lui déconseille de faire le voyage. À la suite de quoi, son épouse et ses quatre enfants viennent à Lisbonne et, avec I'aide de I'oncle maternel, achètent un appartement pour pouvoir s'occuper de lui. En janvier 2006, Alam meurt et sa famille décide de l'enterrer à Lisbonne, dans le carré musulman du cimetière de Lumiar. La famille interrogée sur cette décision explique qu'il devait être enterré le plus vite possible et que par conséquent elle ne voulait pas le transférer au Bangladesh. Alam 
n'avait plus de parents (atyio) au Bangladesh et sa sœur qui vivait à Dhaka, ne voulait pas entendre parler de son frère. Dans ces conditions il était inutile de rapatrier le corps au Bangladesh puisque personne ne pouvait s'en occuper. Sa famille était au Portugal et plus au Bangladesh.

Le gendre et les fils participèrent aux ablutions du cadavre dans la mosquée centrale de Lisbonne. La salat-ul-janazah fut effectuée dans le patio rassemblant une trentaine de Bangladais, parmi lesquels les figures les plus prestigieuses de la "communauté ", des amis et des connaissances de la famille. Ensuite, on referma le cercueil et le corps fut emmené au cimetière de Lumiar. Au cimetière, le corps retiré du cercueil fut enveloppé dans le kaffan, puis déposé dans la tombe, la tête tournée vers la Mecque. La tombe fut recouverte de planche puis recouverte de terre par les employés du cimetière et quelques présents qui récitaient des prières. Ce fut le premier enterrement d'un Bangladais au Portugal.

\section{Conclusion : le lieu de l'enterrement et la « bonne mort »}

Les rituels funéraires constituent des systèmes de circulation de biens entre les vivants et les morts, et contribuent au renforcement du continuum entre les deux mondes : dans le cas guinéen, les défunts ne deviennent pas des esprits protecteurs si les rites n'ont pas été correctement et intégralement pratiqués (Saraiva, 2004a, 2004b et 2008) ; dans le cas des Bangladais, les esprits des défunts ne pourront entrer au paradis sans les prières des vivants (Mapril, 2010).

Pour Bloch (1982: 15) la notion de " bonne mort " est liée à la nécessité humaine de contrôler l'imprévisibilité de la mort biologique, en introduisant des prototypes idéaux de situations où la mort est domestiquée et transformée en un élément d'un ordre répétitif cyclique qui deviendra, en dernière instance, régénération et reproduction de la vie. Se centrant sur différents exemples, en particulier celui des Merina et des Lugbara, Bloch spécifie que, sans enterrement dans la tombe communautaire ou près de la maison ancestrale, non seulement on perd une source potentielle de régénération pour le groupe, mais la mort de l'individu devient une fin. L'emphase placée sur le lieu de repos du corps mort pour qu'il ait une " bonne mort " est évidente. Cette prémisse est présente dans les cas décrits ci-dessus, tant par la concrétisation physique de cette règle - à travers l'envoi du corps lui-même - que par sa transformation symbolique et son dédoublement en diverses actions pouvant surmonter l'impossibilité de le faire matériellement : I'envoi de la valise, les cérémonies réparties entre les deux espaces (guinéen et portugais), le toka chur, pour les Guinéens ; les assemblées de dévotion, les prières et oraisons effectuées à distance, dans le cas des Bangladais. Ce qui est sous-entendu dans ces dynamiques cérémonielles, c'est précisément le lien entre migration, réciprocité, redistribution et " bonne mort ".

L'émigration est fréquemment vécue comme un projet stimulant où I'on recherche prestige et réussite économique. La réussite, quand elle se concrétise, doit être partagée avec les autres parents dans une logique de réciprocité et de co-responsabilité morale (qui implique un lien avec la terre et la famille d'origine) et qui a des conséquences sur les perceptions de la mort et du mourir. Les migrants espèrent que ceux qui sont restés en Guinée ou au Bangladesh s'occuperont d'eux, en accueillant leurs cadavres ou en procédant à des cérémonies nécessaires à la " bonne mort ", car ils se sont sacrifiés pour le bien-être collectif. En même temps, le migrant qui a réussi est dans l'obligation de veiller sur ses parents et ses compatriotes en cherchant à aider ou à parrainer leurs cérémonies funèbres et à contribuer ainsi, par sa réussite, à la " bonne mort " des proches. 
Ce que donnent à voir nos enquêtes, c'est que les "lieux " n'ont pas cessé d'exister dans la mondialisation; ils continuent d'être produits dans le cadre de la vie sociale (Olwig, 2007). Il existe une homologie entre les lieux d'appartenance et la notion de relatedness (Carsten, 2000), qui atteint son oxymoron dans la mort et le mourir. En territorialisant la mort, on célèbre l'union avec un lieu où les gens ont leurs parents et leurs connaissances, union qui représente une victoire sur la division et la séparation que la contemporanéité induit en poussant beaucoup de gens à émigrer. De fait, dans une société de migrants, l'important n'est pas le lieu de naissance, mais le lieu d'enterrement (Ho, 2006) et les transits funèbres entre le Portugal, le Bangladesh et la Guinée-Bissau sont révélateurs de ces dynamiques telluriques.

Pour reprendre les analyses de Bloch sur la bonne mort comme potentiel de régénération pour le groupe qui empêche aussi que la mort d'un individu soit la fin, nous avons voulu montrer la manière dont agit la notion de relatedness dans deux contextes ethnographiques différents et dans des situations de diaspora différenciées. Dans les réseaux transnationaux bangladais et guinéens, il existe une relation intense entre les mondes parallèles - les mondes physiques concrets des contextes nationaux $d^{\prime}$ origine des immigrants et le pays d'accueil - entre lesquels circulent notamment les personnes et les cadavres. Cette circulation est de la responsabilité des migrants, qui remplissent leurs devoirs autant envers les morts qu'envers leurs familles dans l'espace d'origine. Circulent aussi des biens symboliques et des esprits, par l'intermédiaire desquels les vivants remplissent leurs devoirs envers les défunts. Ces dynamiques renvoient une fois encore à la notion de relatedness, dans la mesure où la "communauté " (guinéenne ou bangladaise) existe à l'intersection entre le monde des vivants et celui des morts. Autrement dit, ces "communautés " sont composées de vivants et de morts, et ce que l'on attend des vivants - migrants - c'est d'assurer le maintien de cette continuité bien qu'ils soient loin de leur lieu d'origine. En même temps, cela révèle que la mort d'une personne n'est jamais une fin, et que cette mort est une source de régénération - ici, régénération de la vie même dans les espaces élargis par l'émigration, puisque c'est la mort qui permet la continuité de l'ensemble des relations avec le lieu d'origine.

\section{Références bibliographiques}

Ariés Philippe (1989) Sobre a História da Morte no Ocidente desde a Idade Média, Lisboa, Teorema, $312 \mathrm{p}$.

Baganha Maria, Malheiros Jorge e Ferrão João (1999) Os imigrantes e o mercado de trabalho: o caso português, Revista Análise Social, 150, pp. 147-173.

Basch Linda Schiller Nina and Blanc-Szanton Cristina (1997) Nations Unbound: Transnational Projects, Postcolonial Predicaments and Deterritorialized Nation-States, Amsterdam, Gordon and Breach Publishers, $344 \mathrm{p}$.

Basch Linda, Schiller Nina and Blanc-Szanton Cristina (1992), Transnationalism: a new analytic framework for understanding migration, Annals of the New York Academy of Sciences, 645, pp. 1-24.

Bloch Maurice (1982) Death, women and power, in Maurice Bloch and Jonathan Parry Eds., Death and the Regeneration of Life, Cambridge, Cambridge University Press, pp. 211-230. 
Bloch Maurice (1971) Placing the Dead:Tombs, Ancestral Villages, and Kinship Organization in Madagascar, Londres, Seminar Press, $241 \mathrm{p}$.

Bloch Maurice and Parry Jonathan (Eds.) (1982) Death and the Regeneration of Life, Cambridge, Cambridge University Press, 248 p.

Bordonaro Lorenzo e Pussetti Chiara (2006) Da utopia da migração à nostalgia dos migrantes: percursos migratórios entre Bubaque (Guiné-Bissau) e Lisboa, in Antónia Lima e R. Sarró Eds., Terrenos Metropolitanos. Ensaios sobre produção etnográfica, Lisboa, Imprensa de Ciências Sociais, pp. 125-153.

Carsten Janet (Ed.) (2000) Cultures of Relatedness: New approaches to the study of kinship, Cambridge, Cambridge University Press, 215 p.

De Boeck Philippe (2008) Danças com os mortos: os jovens em Kinshasa e a ordem da desordem, présentée lors de la conference Gulbenkian, Distancia e Proximidade, Lisboa, 27 et 28 octobre.

Douglass William (1969) Death in Murelaga. Funeral Ritual in a Spanish Basque Village, Seattle, University of Washington Press, 244 p.

Eaton Richard (1993) The Rise of Islam and the Bengal Frontier, 1204-1760, Berkeley, University of California Press, 359 p.

Einarsdottir Jonina (2000) "Tired of Weeping". Child Death and Mourning among Papel Mothers in Guinea-Bissau, Stockholm, Stockholm University, 250 p.

Gable Eric (2006) The Funeral and modernity in Manjaco, Cultural Anthropology, 21 (3), pp. 385-415.

Garbin David (2004) Migrations, Territoires Diasporiques et Politiques Identitaires : Bengalis musulmans entre "Banglatown " et Sylhet, Thèse de doctorat, Tours, Université François Rabelais.

Gardner Katy (2002) Age, Narrative and Migration: The Life Course and Life Histories of Bengali Elders in London, London, Berg Publishers, 224 p.

Gardner Katy (1998) Death, burial and bereavement amongst Bengali Muslims in Tower Hamlets, Journal of Ethnic and Migration Studies, 24 (3), pp. 507-521.

Gardner Katy (1995) Global Migrants, Local Lives: Travel and Transformation in Rural Bangladesh, Oxford, Oxford University Press, 316 p.

Gardner Katy (1993) Desh bidesh: Sylheti images of home and away, Man, 28 (1), pp. 1-15.

Geertz Clifford (1996) "Afterword", in Steven Feld and Keith Basso Eds., Senses of Place, Santa Fé, School of American Research Press, pp. 259-262.

Geschiere Peter (2005) Funerals and belonging: different patterns in South Cameroon, Africa Studies Review, 48 (2), pp. 45-64.

Ho Engseng (2006) The Graves of Tarim: Genealogy and Mobility across the Indian Ocean, Berkeley, University of California Press, 379 p. 
Jao Mamadu (1995) A questão da etnicidade e a origem étnica dos Mancanhas, Soronda. Revista de Estudos Guineenses, 20, pp. 19-31.

King Russel, Lazaridis Gabriella and Tsardanidis Charalampos (Eds.) (2000) Eldorado or Fortress? Migration in Southern Europe, London, MacMillan Press, 368 p.

Knights Melanie (1997) Migrants as networkers: the economics of Bangladeshi migration to Rome, in Russel King and Richard Black Eds., Southern Europe and the New Immigrations, Brighton, Sussex Academic Press, pp. 113-137.

Knights Melanie (1996) Bangladeshi immigrants in Italy: from geopolitics to micropolitics, Trans. Inst. Br. Geogr., 21, pp. 105-123.

Kopytoff Igor (1971) Ancestors as Elders in Africa, Africa, 41 (2), pp. 129-142.

Kotalová Jitka (1993) Belonging to Others: Cultural Construction of Womenhood in a Village in Bangladesh, Dhaka, University Press Limited, 252 p.

Lestage Françoise (2008) Apuntes relativos a la repatriación de los cuerpos de los mexicanos fallecidos en Estados Unidos, Migraciones Internacionales, 4 (4), pp. 217-228.

Machado Fernando Luis (2002) Contrastes e Continuidades. Migração, Etnicidade e Integração dos Guineenses em Portugal, Oeiras, Celta, 464 p.

Malheiros Jorge (1996) Imigrantes na Região de Lisboa: Os anos da Mudança, Lisboa, Edições Colibri, 238 p.

Mapril José (2007) Os sonhos da «modernidade»: migrações globais e consumos entre Lisboa e Dhaka, in Renato Carmo, Ruy Blanes e Daniel Melo Eds., A Globalização no Divã, Lisboa, Tinta da China, pp. 65-88.

Mapril José (2005) Bangla masdjid: Islão e bengalidade entre os bangladechianos em Lisboa, Análise Social, 173, pp. 851-873

Metcalf Peter (1985) Death be not strange, in Arthur Lehman and James Myers Eds., Magic, Witchcraft and Religion. An Anthropological Study of the Supernatural, Mountain View, Mayfield Publishing Company.

Olwig Karen (2007) Caribbean Journeys: An Ethnography of Migration and Home in Three Family Networks, Durham, Duke University Press, 336 p.

Pina Cabral João (1984) A morte na Antropologia Social, Análise Social, 20, pp. 349-356.

Quintino Celeste (2004) Migrações e Etnicidade em Terrenos Portugueses. Guineenses : Estratégias de Invenção de uma Comunidade, Lisboa, Universidade Técnica de Lisboa, Instituto Superior de Ciências Sociais e Politicas, 553 p.

Saraiva Clara (2008) Transnational migrants and transnational spirits: an African religion in Lisbon, Journal of Ethnic and Migration Studies, 32 (4), pp. 253-269.

Saraiva Clara (2004a) Embalming, sprinkling and wrapping bodies. Death ways in America, Portugal and Guinea-Bissau: a cross-cultural study, Symposia. Journal for Studies in Ethnology and Anthropology, 2004, pp. 97-119. 
Saraiva Clara (2004b) Rituais funerários e concepções da morte na etnia Papel da GuinéBissau-Parte II, Soronda, Revista de estudos Guineenses nova série, 8, pp. 109-133.

Saraiva Clara (2003) Rituais funerários e concepções da morte na etnia Papel da GuinéBissau-Parte I, Soronda, Revista de Estudos Guineenses nova série, 6, pp. 179-210.

Sarró Ramon (2009) The politics of religious change on the Upper Guinea coast. Iconoclasm done and undone, Edinburgh, Edinburgh University Press, 264 p.

Siddiqui Tasneem (2001) Transcending Boundaries: Labour Migration of Women from Bangladesh, Dhaka, University Press Limited, 208 p.

Tiesler Nina (2000) Muçulmanos na margem: A nova presença islâmica em Portugal, Sociologia : Problemas e Práticas, 34, pp. 117-144.

Thomas Louis-Vincent (1982) La mort africaine, Paris, Payot, 272 p.

Thomas Louis-Vincent (1975) Anthropologie de la mort, Paris, Payot, 540 p.

Vakil Abdoolkarim (2003) Muslims in Portugal: History, historiography, citizenship, EuroClio Bulletin, 18, pp. 9-13

Vovelle Michel (1983) La mort et l'Occident de 1300 à nos jours, Paris, Gallimard, 793 p. 


\section{Clara Saraiva et José Mapril}

\section{Le lieu de la " bonne mort " pour les migrants guinéens et bangladais au Portugal}

Les dimensions transnationales des migrations ont pris une importance théorique et ethnographique croissante. Les liens et mobilités qu'elles supposent sont souvent accompagnés par la construction sociale et symbolique des espaces d'appartenance. Dans cet article, nous montrons que la mort est une bonne métaphore pour penser cette production des lieux et des espaces d'appartenance dans des contextes transnationaux. La mort implique la circulation d'univers symboliques, dans lesquels la notion de processus est fondamentale, en particulier dans le maintien de la relation avec le lieu d'origine. Ces arguments sont explorés à travers deux études de cas : les migrants en provenance du Bangladesh et ceux de Guinée-Bissau au Portugal.

\section{... The Place of "Good Death" for the Migrants from Bangladesh and from Guinea-Bissau in Portugal}

The transnational dimensions of migration have grown in importance, both from a theoretical and an ethnographical point of view. Such ties and mobilities are frequently accompanied by the social and symbolic construction of spaces of belonging. In this text we want to show how death is a good metaphor to think about the production of places and spaces of belonging and relating in several transnational contexts. Death implies the circulation of symbolic universes in which the notion of process is extremely important, especially in what concerns the maintenance of the relationship with the space of origin. Such arguments will be explored through two case studies: the migrants from Bangladesh and the ones from Guinea-Bissau in Portugal.

\section{El lugar de la ubuena muerte" para los migrantes guieneses y bangladeshís en Portugal}

Las dimensiones transnacionales de las migraciones han adquirido una creciente importancia teórica y etnográfica. Tales vínculos y movilidades son acompañados a menudo por la construcción social y simbólica de los espacios de pertenencia. Este artículo pretende mostrar que la muerte es una buena metáfora para reflexionar sobre esta producción de lugares y espacios de pertenencia en contextos transnacionales. La muerte implica la circulación de los universos simbólicos, en la que la noción de proceso es de suma importancia, en particular en el mantenimiento de la relación con el espacio de origen. Estos argumentos serán explorados a través de dos estudios de caso: los inmigrantes de Bangladesh y los de Guinea-Bissau en Portugal.

\section{O lugar da «boa norte» entre imigrantes do Bangladeche e da Guiné-Bissau em Portugal}

As dimensões transnacionais dos fenómenos migratórios têm assumido uma crescente importância teórica e etnográfica. Constata-se que tais laços e mobilidades são frequentemente acompanhados pela construção social e simbólica de espaços de pertença. Neste texto queremos mostrar que a morte é uma boa metáfora para pensar esta produção de lugares e espaços de pertença em contextos transnacionais. A morte implica a circulação de universos simbólicos, em que a noção de processo é extremamente importante, sobretudo na manutenção da relação com o espaço de origem. Tais argumentos serão explorados através de dois estudos de caso: os migrantes do Bangladeche e da GuinéBissau em Portugal. 